\title{
Orientation asymmetry in the flanker task
}

\author{
ASHER COHEN and RACHEL E. SHOUP \\ Indiana University, Bloomington, Indiana
}

\begin{abstract}
Experiments 1 and 2 of this study show that when the target is either a vertical or a horizontal line, diagonal-line flankers tilted $45^{\circ}$ either to the right or to the left have the same effect as do incongruent flankers. When the target is a diagonal line tilted either to the right or to the left, vertical- or horizontal-line flankers do not have the same effect as do incongruent flankers. Experiment 3 demonstrates that this asymmetry is not caused by the temporal-dynamic aspects of the processing. Together, these experiments suggest that there is an asymmetrical relation between diagonal lines and either vertical or horizontal lines outside of the central focus of attention. Experiment 4 shows that despite this asymmetry in the flanker task, visual search for a vertical- or a horizontal-line target among diagonal-line distractors is not affected by the number of distractors. Possible explanations of this phenomenon are discussed.
\end{abstract}

The purpose of this article is to demonstrate an asymmetry in orientation between diagonal lines and either vertical or horizontal lines when these lines are presented outside the central focus of attention. We use the flanker task, first introduced by B. A. Eriksen and C. A. Eriksen (1974), to demonstrate this asymmetry.

The flanker task was originally designed to contrast early-selection theories of attention (e.g., Broadbent, 1958), according to which the processing of stimuli outside the focus of attention is confined to rudimentary physical properties, and late-selection theories (e.g., Deutsch \& Deutsch, 1963), according to which stimuli outside the focus of attention are fully identified. In a typical task, subjects are instructed to make a specific response to the appearance of one target (e.g., the letter X) and to make a different response to the appearance of another target (e.g., the letter $\mathrm{O}$ ). The target is typically presented in the center of the visual field and is flanked by stimuli (one or more) on each side. Because the target location is known in advance, and because the demand characteristics of the task are to focus on the target and ignore the flankers, it is assumed that subjects try to focus their attention exclusively on the target. The key question in this paradigm is whether the identity of the flanking stimuli affects responses to the targets. The flankers may be either neutral (e.g., the letter F), congruent (e.g., both target and flankers are $\mathrm{O}$ or both are $\mathrm{X}$ ), or incongruent (e.g., the target is $X$ and the flankers are $O$ or vice versa).

Most studies using the flanker task have found that both flanker congruency and spatial distance between the target and flankers influence subjects' responses to the tar-

This study was supported by NSF Grant BNS-9012471. We would like to thank Jim Craig, Charles W. Eriksen, Rich Ivry, Rich Shiffrin, and three anonymous reviewers for their helpful discussions and comments. We also thank Ingrid Janse for her assistance in conducting the experiments. Correspondence concerning this article should be addressed to Asher Cohen, Department of Psychology, Hebrew University, Jerusalem, Israel (e-mail: msasher@pluto.cc.huji.ac.il or ascohen@ucs. indiana.edu). get. Reaction times to targets with congruent flankers were faster than those to targets with incongruent flankers (e.g., B. A. Eriksen \& C. W. Eriksen, 1974; C. W. Eriksen \& B. A. Eriksen, 1979; C. W. Eriksen \& Schultz, 1979; Harms \& Bundesen, 1983; Miller, 1991). Studies in which the spatial separation of target and flankers was manipulated showed that the effect of congruency was smaller with increased separation but was still present even at relatively large distances (e.g., B. A. Eriksen \& C. W. Eriksen, 1974; Miller, 1991).

Much evidence suggests that both perceptual interaction between the target and flankers and processes concerned with response selection influence the congruency effect. Given that congruency is defined by the response properties of the stimuli rather than by the perceptual similarity between the stimuli, the very existence of the congruency effect suggests that response competition is an important factor. Other studies support this observation more directly. C. W. Eriksen and B. A. Eriksen (1979) asked their subjects to make one response to either one of two different characters ( $\mathrm{H}$ and $\mathrm{C}$ ) and to make a second response to either one of two other characters ( $K$ and $S$ ). They found that when the target and flankers were composed of different characters, the response of the subjects was faster when the flankers and the target belonged to the same response set (e.g., $\mathrm{H}$ flanked by $\mathrm{Cs}$ ) than when the target and flankers belonged to two different response sets (e.g., $\mathrm{H}$ flanked by Ks). Because perceptual similarity was no greater in the congruent condition, this finding indicates that flanker interference occurs at the response level (see also C. W. Eriksen \& Schultz, 1979; Grice \& Gwynne, 1985). Coles, Gratton, Bashore, C. W. Eriksen, and Donchin (1985), using several psychophysiological measures, also showed that at least part of the congruency effect is due to response competition. Miller (1987) showed that neutral letters that are correlated with a particular response act as congruent stimuli for this response.

Several studies have shown that physical characteristics of the stimuli also contribute to the congruency effect. 
B. A. Eriksen and C. W. Eriksen (1974) found that neutral flankers that were physically similar to incongruent flankers caused a slower response than did neutral flankers that were physically similar to congruent flankers. Other studies have shown that when two characters are assigned to the same response set, subjects respond faster when the flankers are both congruent and identical to the target than when the flankers are congruent but not identical to the target (C. W. Eriksen \& B. A. Eriksen, 1979; C. W. Eriksen \& Schultz, 1979; Grice \& Gwynne, 1985). Yeh and C. W. Eriksen (1984) found that physical similarity between the target and the flankers influences the congruency effect more than does name similarity. Several studies (e.g., Harms \& Bundesen, 1983; Kramer \& Jacobson, 1991) demonstrated that perceptual grouping principles also affect the degree of interaction between the target and the flankers.

The interpretation of these results with respect to earlyversus late-selection theories of attention has proven to be complicated. Although the results of the flanker task generally seem to support late-selection theories of attention (see Coles et al., 1985; C. W. Eriksen \& Schultz, 1979; Miller, 1987, 1991), alternative explanations may still be possible (Yantis \& Johnston, 1990).

However, because the flanker task is sensitive to the structural relation (such as perceptual similarity) between flankers and targets, it may enable us to assess the nature of this relation among visual stimuli. The logic here is as follows. Previous studies have shown that when flankers are neutral, their effect on reaction time is directly related to their structural relation to the targets. For example, neutral stimuli that are physically similar to one of the targets also show similar congruency effects (B. A. Eriksen \& C. W. Eriksen, 1974). That is, subjects are faster when these neutral stimuli flank the target to which they are similar than when these stimuli flank the other target. Therefore, it may be possible to assess the nature of the structural relation of neutral stimuli to the targets by their influence as flankers on reaction times to the target. For example, subjects may be required to make one response to Stimulus $\mathrm{A}$ and another response to Stimulus B. Stimulus $\mathrm{C}$ does not require any response and is therefore neutral. The degree of congruency of Stimulus $C$ with the Targets $A$ and $B$ may indicate the structural relation among these stimuli.

In this article, we use this logic to demonstrate an asymmetry in the structural relation between diagonal lines and either vertical or horizontal lines. Specifically, we show that neutral diagonal-line flankers that are tilted $45^{\circ}$ are incongruent with both vertical- and horizontal-line targets. In contrast, neither vertical- nor horizontal-line flankers appear to be incongruent with diagonal-line targets. Experiments 1 and 2 document this phenomenon. Experiment 3 examines and rejects an alternative explanation that is not based on the nature of the structural relation between line orientations. Experiment 4 investigates whether the asymmetry between diagonal and vertical/ horizontal lines occurs in a visual search paradigm.

\section{EXPERIMENT 1}

This experiment was originally designed to further examine a study reported by Driver and Baylis (1989). ${ }^{1}$ The arrangement of congruent, incongruent, and neutral stimuli was similar to the design of Driver and Baylis, except that the stimuli in our experiments all remained stationary. On each trial, the target appeared in the middle of the screen and was preceded by an asterisk that clearly marked its location. The target was always flanked by two stimuli on each side. The five stimuli appeared along the central horizontal line of the screen and were equidistant from one another. The rightmost and leftmost stimuli (the "far" flankers) were always identical to each other, as were the flankers located immediately to the right and left of the target (the "near" flankers).

Two groups of subjects participated in this experiment. The subjects in the "vertical/horizontal" task were required to make one response to the appearance of a vertical line and another response to the appearance of a horizontal line. The neutral stimuli in this task consisted of diagonal lines tilted $45^{\circ}$ either to the right or to the left of the vertical meridian. The subjects in the "diagonal" task responded to lines tilted $45^{\circ}$ to the right by pressing one key mounted on a response board, and they responded to lines tilted $45^{\circ}$ to the left by pressing a second key. The neutral stimuli for this group were either vertical or horizontal lines.

\section{Method}

Subjects. Twenty-four undergraduate students from Indiana University participated in this experiment in partial fulfillment of a course requirement. Twelve subjects performed the vertical/horizontal task; the other 12 subjects performed the diagonal task. All of the subjects had normal or corrected-to-normal vision.

Apparatus and Stimuli. The stimuli were presented on an NEC MultiSynch SD color monitor that was controlled by a CompuAdd microcomputer. The subjects were tested in a dimly lit room and viewed the display from a distance of $125 \mathrm{~cm}$ with the aid of a chinrest. All of the stimuli consisted of achromatic lines. The length of all lines subtended approximately $0.48^{\circ}$. The five stimuli shown on each display were centered around the middle of the screen and were equidistant from one another. The center-to-center distance between two adjacent stimuli was approximately $0.6^{\circ}$. Thus, the distance between the target and the far flankers was $1.2^{\circ}$. The stimuli were presented against a black background $\left(0 \mathrm{~cd} / \mathrm{m}^{2}\right)$ at a luminance of $76.6 \mathrm{~cd} / \mathrm{m}^{2}$.

Design. The relation between the target and the flankers was manipulated to create five conditions. In the "near congruent" condition, the near flankers were identical to the target, and the far flankers were neutral. In the "far congruent" condition, the far flankers were identical to the target, and the near flankers were neutral. In the "baseline" condition, all four flankers were neutral. In the "near incongruent" condition, the near flankers were identical to the alternative target (i.e., the target not shown in the display), and the far flankers were neutral. In the "far incongruent", condition, the far flankers were identical to the alternative target, and the near flankers were neutral. Note that at least one pair of neutral flankers appeared in every condition. An illustration of the stimuli in the different conditions is shown in Figure 1.

The subjects first received a short demonstration of 9 trials. Following this demonstration, the subjects performed a practice block 


\section{VERTICAL/HORIZONTAL TASK}

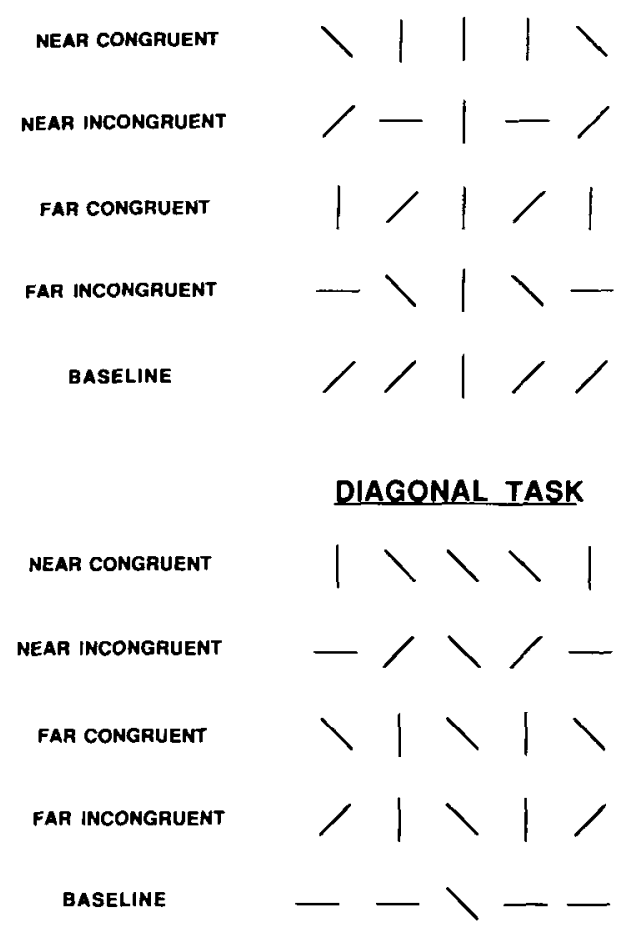

Figure 1. Examples of the stimuli used in the five congruency conditions of Experiment 1.

of 90 trials, and eight experimental blocks of 90 trials each, for a total of 720 experimental trials. Each block consisted of 10 trials from the baseline condition and 20 trials for each of the four remaining congruency conditions. The order of the trials within each block was selected randomly.

Procedure. At the beginning of each trial, an asterisk serving as both a fixation point and a marker of the location of the target was presented in the center of the visual screen. After $500 \mathrm{msec}$, the asterisk was replaced by the display of five stimuli. The display remained visible until the subject pressed one of two keys mounted on a response board interfaced with the computer. In the diagonal task, the subjects had to press a right response key if the target was tilted to the right and a left response key if the target was tilted to the left. In the vertical/horizontal task, the subjects pushed the right key if the target was a vertical line and the left key if the target was a horizontal line. The subjects were instructed to respond as fast as they could while minimizing their mistakes. An error message was presented on the screen for $500 \mathrm{msec}$ following incorrect responses. The screen went blank immediately following the subject's correct response until the appearance of the asterisk of the next trial. The intertrial interval (ITI) was $1,000 \mathrm{msec}$.

\section{Results and Discussion}

The proportion of errors in all of the experiments reported in this study had the same pattern as did the reaction time (RT) results. Thus, the differences among the RTs of the different conditions in the experiments cannot be attributed to a speed-accuracy tradeoff. We reported only the RT results.

The mean RTs of the subjects in the vertical/horizontal task as a function of the five different congruency conditions are shown in the top panel of Figure 2. The mean
RTs of the subjects in the diagonal task are shown in the bottom panel. Note that we separated the two congruent and two incongruent conditions on the basis of their distance from the target. We included only correct responses. In addition, for each subject, we eliminated responses that were 3 standard deviations (SDs) above his/her mean.

As can be seen in the figure, there were no differences among the five congruency conditions in the vertical/ horizontal task. By contrast, the congruency manipulation in the diagonal task produced typical results for the flanker task. That is, RT for congruent conditions was faster than RT for incongruent conditions. RT to targets with neutral flankers, although intermediate, was much closer to that obtained for the congruent conditions. As the distance between the target and the flankers increased, the congruency effect decreased but was still clearly present.

These observations were confirmed by a statistical analysis of the data. The results were submitted to a 2 (task) $\times 5$ (congruency) mixed design analysis of variance (ANOVA). The effect of task was not significant $[F(1,22)=1.4, p<.05]$. The effect of congruency was significant $[F(4,88)=31.3, p<.05]$. Most importantly, the task $\times$ congruency interaction was significant $[F(4,88)=23.3, p<.05]$. Additional contrasts revealed
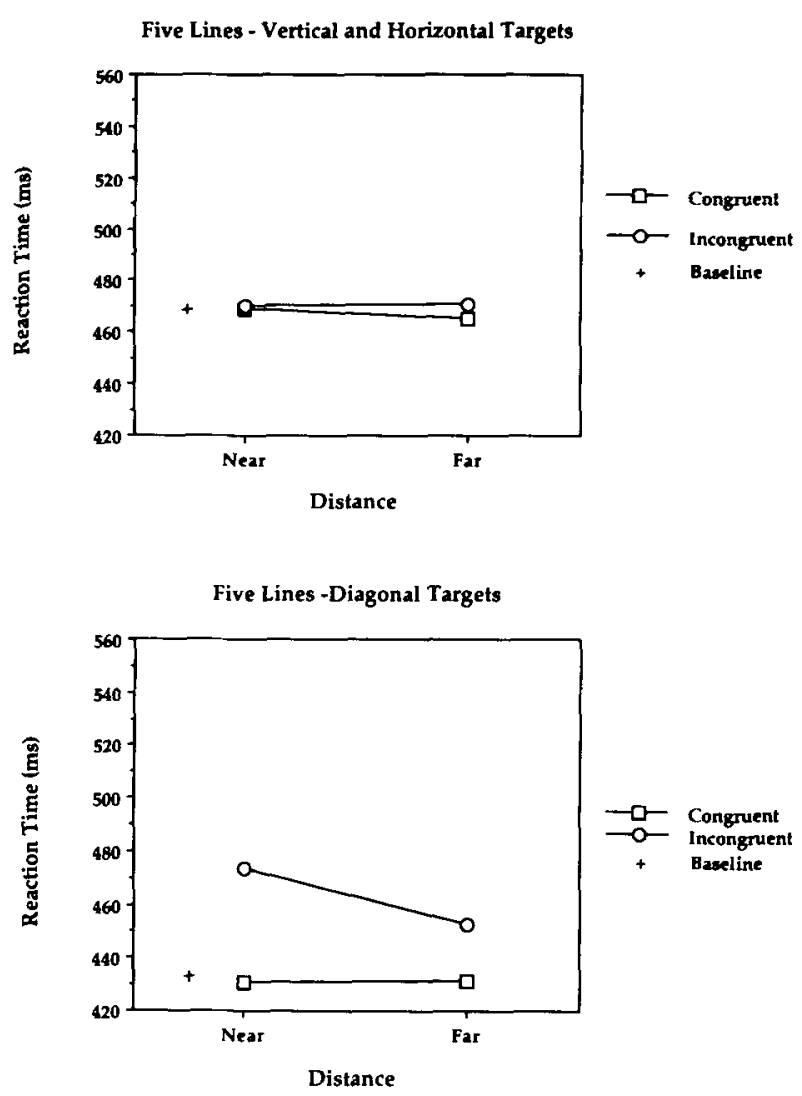

Figure 2. Mean RTs for the five congruency conditions of Experiment 1. The top panel shows the RTs of the vertical/horizontal task, and the bottom panel shows the RTs of the diagonal task. 
that there was no difference between any of the congruent conditions in the vertical/horizontal task. In the diagonal task, the baseline was not significantly different from the congruent conditions $[F(1,11)=1.0, p>.05]$ but was different from the incongruent conditions $[F(1,11)=$ $40.0, p<.05]$. The difference between congruent and incongruent flankers was significant for both the nearflanker conditions $[F(1,11)=77.1, p<.05]$ and the farflanker conditions $[F(1,11)=47.4, p<.05]$. The reduced congruency effect with distance is implied by a significant difference between the near-incongruent and the far-incongruent conditions $[F(1,11)=72.4, p<.05]$.

The results of the diagonal task were consistent with previous flanker-task studies (e.g., B. A. Eriksen \& C. W. Eriksen, 1974; Miller, 1991). There was a pronounced congruency effect for the near conditions, which was weaker but still present for the far conditions. The baseline condition was intermediate but much closer to the congruent conditions. Unlike typical results with the flanker task, no congruency effect was obtained in the vertical/horizontal task.

One clue to the findings of this experiment lies in the method used here. An important aspect of our method was that neutral flankers were present in every single trial. It is possible that neutral diagonal-line flankers have the same effect as do incongruent flankers when the target consists of either vertical or horizontal lines. Because diagonal lines were present in every trial, there may have existed a degree of incongruency in all trials that caused similar responses in all conditions. By contrast, verticaland horizontal-line flankers do not affect diagonal-line targets in the way incongruent flankers do, and thus the typical congruency effect was obtained.

One immediate prediction of our hypothesis is that we should find a congruency effect in the vertical/horizontal task if diagonal lines are not present in all trials. This prediction was tested in Experiment 2A. Experiments $2 \mathrm{~B}$ and $2 \mathrm{C}$ examine alternative interpretations of Experiment $2 \mathrm{~A}$.

\section{EXPERIMENTS 2A, 2B, AND 2C}

The method used in this experiment is similar to the one employed in Experiment 1, with three differences. First, three, rather than five, stimuli were presented on each trial. In Experiment 2A, the two flankers could be congruent, incongruent, or neutral. In Experiment 2B, there were no neutral flankers, and the two flankers could be either congruent or incongruent. The flankers in Experiment $2 \mathrm{C}$ (done only with the vertical/horizontal task) consisted only of neutral diagonal-line flankers. Second, the spatial distance between the target and the flankers was manipulated such that it was either identical to the distance used between the near flankers and the target of Experiment 1 or identical to the distance between the far flankers and the target in Experiment 1. Third, the size of the stimuli was manipulated. There were two different lengths for each of the possible target orientations. ${ }^{2}$

\section{Experiment 2A}

Our prediction for the diagonal task is simple. The results should be similar to those obtained in Experiment 1 . In contrast, the results for the vertical/horizontal version of the present experiment should be different from those obtained with this task in Experiment 1. As in all flanker tasks, we should find a faster response in the congruent conditions than in the incongruent conditions. According to our hypothesis, the neutral diagonal-line flankers have the same effect as do incongruent flankers for both vertical- and horizontal-line targets. Therefore, the RT for the baseline condition with diagonal flankers should be similar to the RT for the incongruent condition.

\section{Method}

Subjects. Twenty-four subjects, recruited from the same pool as in Experiment 1, participated in this experiment. Half of the subjects performed the vertical/horizontal task, and half performed the diagonal task.

Apparatus and Stimuli. Both the stimuli and apparatus were identical to those of Experiment 1 except for the size of the stimuli. There were two different lengths for each of the possible target orientations. For the vertical/horizontal task, the target subtended approximately $0.41^{\circ}$ and $0.18^{\circ}$ of visual angle. The neutral diagonal line occurred in only one size, approximately $0.28^{\circ}$. The targets for the diagonal task consisted of achromatic lines tilted $45^{\circ}$ either to the right or left. The two sizes used in this condition subtended approximately $0.55^{\circ}$ and $0.23^{\circ}$. The length of the neutral lines (both vertical and horizontal) subtended approximately $0.41^{\circ}$. The centerto-center distance between two adjacent stimuli in the near condition was approximately $0.6^{\circ}$. The distance between the target and the flankers in the far condition was $1.2^{\circ}$

Design. The design of 3 (congruency) $\times 2$ (distance) mentioned above created six different conditions that were run within each block. In addition, we manipulated the size of the stimuli in the congruent and incongruent conditions. The size of the target and flankers was identical in half of the trials of these conditions and different in the other half.

Following a 10-trial demonstration of the task, the subjects performed one practice block of 100 trials and eight experimental blocks of 100 trials each, for a total of 800 experimental trials. Each block consisted of 10 trials of the near-baseline condition, 10 trials of the far-baseline condition, and 20 trials each for the near-congruent, far-congruent, near-incongruent, and far-incongruent conditions. The size of the target and flankers was identical in half of these trials and different in the other half. The order of the trials within a block was random.

Procedure. The procedure was identical to that of Experiment 1.

\section{Results and Discussion}

The manipulation of size did not interact with the congruency effect in this experiment. Thus, we combined the results of the same-size and different-size stimuli in each of the congruency conditions.

The mean RTs to the vertical/horizontal task are shown on the top panel of Figure 3, and the mean RTs to the diagonal lines are shown on the bottom panel. As in Experiment 1 , for each subject, we eliminated from the analysis both incorrect responses and responses that were slower than $3 S D$ s above the mean. The results confirmed the predictions of our hypothesis. The diagonal task produced results very similar to those obtained in Exper- 
Three Lines - Vertical and Horizontal Targets

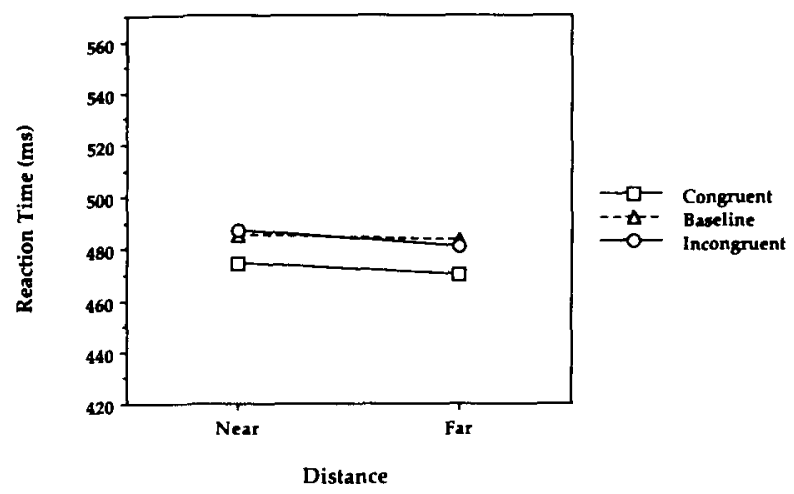

Three Lines - Diagonal Targets

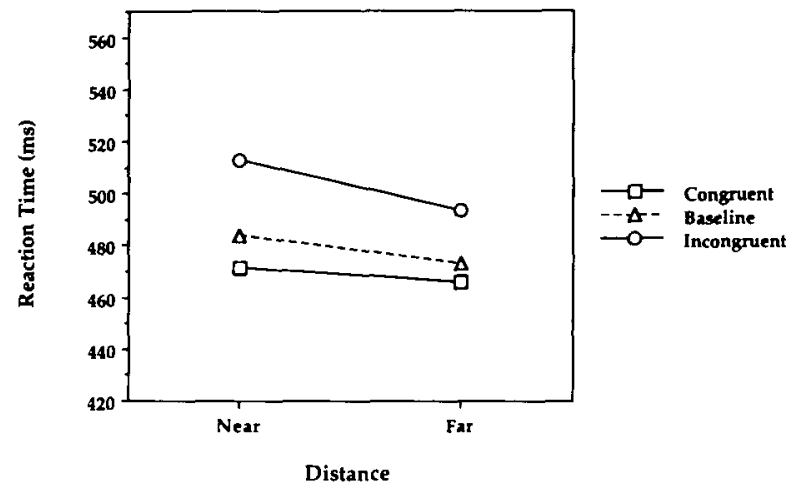

Figure 3. Mean RTs for the six congruency conditions of Experiment $2 A$. The top panel shows the RTs of the vertical/horizontal task, and the bottom panel shows the RTs of the diagonal task.

iment 1. The subjects in this task showed a marked congruency effect. The effect was smaller in the far conditions but was still clearly present. The baseline conditions produced intermediate results that were closer to those obtained in the congruent conditions. As we predicted, for the vertical/horizontal task, the results were different from those of Experiment 1. The subjects in the present experiment were faster in the congruent conditions than in the incongruent conditions. Reaction times for the baseline conditions resembled those for the incongruent conditions.

Statistical analyses of the data supported these observations. A 2 (task: diagonal, vertical/horizontal) $\times 3$ (congruency: congruent, incongruent, neutral) $\times 2$ (distance: near, far) mixed design ANOVA was performed. The main effect of task was not significant $[F(1,22)<1]$. The main effects of congruency $[F(2,44)=57.1]$ and distance $[F(1,22)=31.9]$ were significant at $p<.05$, as were the congruency $\times$ distance interaction $[F(2,44)=3.9]$ and the distance $\times$ task interaction $[F(1,22)=7.8]$. Most importantly, the congruency $\times$ task interaction was highly significant $[F(2,44)=20.5]$. Subsequent contrasts revealed that for the diagonal task, the congruency effect was significant at $p<.05$ at both the near and far dis- tances. The baseline condition differed significantly from both incongruent conditions and congruent conditions. The contrasts of the vertical/horizontal task revealed that, as predicted, the baseline conditions were significantly different from the congruent conditions but not from the incongruent conditions.

Although we obtained a reliable congruency effect in the vertical/horizontal task, the effect was smaller in this task than in the diagonal task. This finding was not predicted by our hypothesis. A possible explanation for this smaller effect is that the presence of the neutral diagonal lines in some of the trials is disruptive in the vertical/ horizontal task and makes the subjects more conservative in their response. One possible reason for this disruption is that, given that the diagonal neutral lines produce effects similar to those of incongruent flankers, the proportion of trials with response competition is larger in this task. Experiment 2B was conducted to examine this possibility.

\section{Experiment 2B}

The only difference between this experiment and Experiment $2 \mathrm{~A}$ is that we eliminated the baseline conditions. Thus, each task consisted only of trials with congruent or incongruent flankers.

\section{Method}

Subjects. Twenty-four subjects, recruited from the same pool as in previous experiments, participated in this experiment. Half of the subjects performed the vertical/horizontal task, and half performed the diagonal task.

Apparatus and Stimuli. The apparatus and stimuli were the same as in Experiment 2A.

Design. The design was the same as in Experiment 2A, except that the neutral flankers were not used in this experiment.

\section{Results and Discussion}

The results of this experiment are shown in Figure 4. The top panel shows the results for the vertical/horizontal task. The bottom figure shows the results for the diagonal task. As can be seen in the figure, the results are quite similar to those obtained in our previous experiments. Statistical analyses of the data supported these observations. A 2 (task: diagonal, vertical/horizontal) $\times 2$ (congruency: congruent, incongruent) $\times 2$ (distance: near, far) mixed design ANOVA was performed. The effect of task was not significant $[F(1,22)=2.0, p<.05]$. The effects of congruency $[F(1,22)=101.9]$ and distance $[F(1,22)=$ 8.4] were significant at $p<.05$. The congruency $\times$ task interaction was also significant $[F(1,22)=26.9, p<$ $.05]$. Separate contrasts revealed that the congruence effect was significant for both the diagonal and the vertical/horizontal tasks. These results show that the congruency effect is present in both tasks but is weaker in the vertical/horizontal task.

We are not sure why the congruency effect is weaker in the vertical/horizontal task. More research is needed to account for these findings. The results of Experiment 2B, however, suggest that the difference in the degree of congruency obtained between the two tasks in Experi- 
Three Lines - Vertical and Horizontal Targets - No Baseline

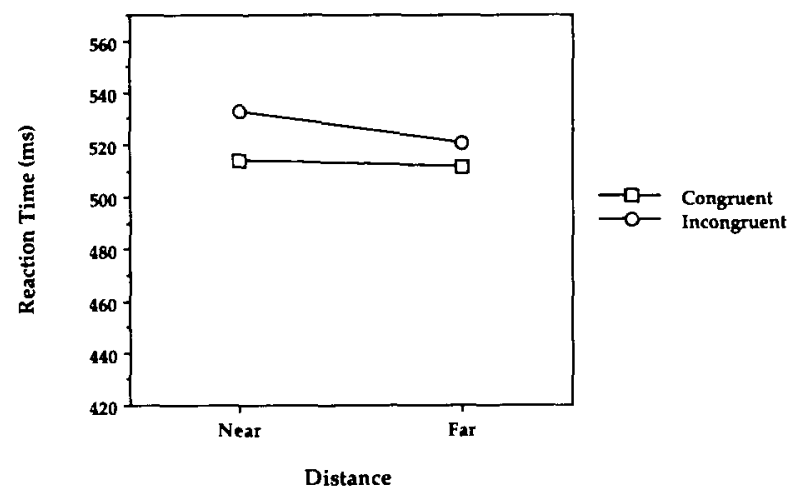

Three Lines - Diagonal Targets - No Baseline

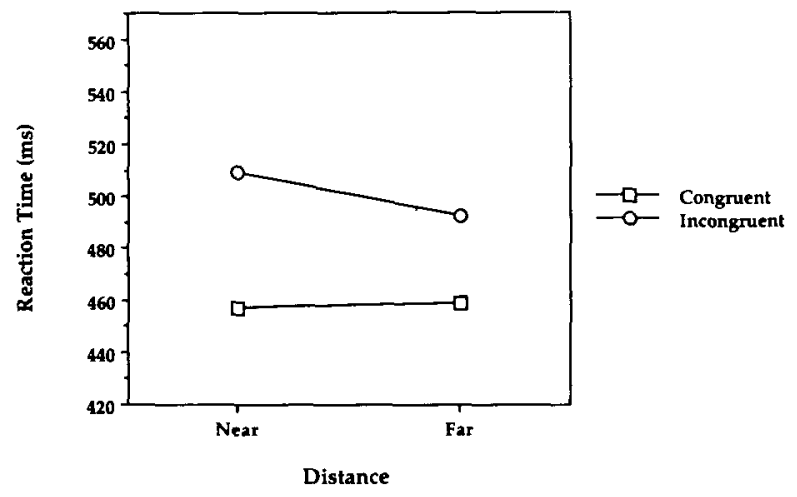

Figure 4. Mean RTs for the four congruency conditions of Experiment 2B. The top panel shows the RTs of the vertical/horizontal task, and the bottom panel shows the RTs of the diagonal task.

ment $2 \mathrm{~A}$ is not due to our specific method but rather to the nature of the stimuli used.

Another explanation of the effect of neutral diagonalline flankers on vertical- and horizontal-line targets is that the presence of diagonal lines exerts some sort of responsecompatibility effect on the subjects. It is possible that diagonal-line flankers tilted to the right induce a response bias with the right response key and that diagonal-line flankers tilted to the left induce a response bias with the left response key. Such a response-compatibility effect may mask the typical congruency effects obtained with vertical- and horizontal-line targets. To the extent that this interpretation is correct, there should be a faster response to a target that requires a right-key response when the flankers consist of diagonal lines tilted to the right than when the flankers consist of diagonal lines tilted to the left. The effect should be reversed when the target requires a left-key response. Experiment $2 \mathrm{C}$ examined this possibility.

\section{Experiment 2C}

The vertical/horizontal task of Experiment $2 \mathrm{~A}$ was used here, with an important change. On half of the trials for each target, the flankers were diagonal lines tilted to the right. On the other half of the trials, the flankers were diagonal lines tilted to the left. The vertical- and horizontal-line flankers were eliminated.

\footnotetext{
Method

Subjects. Twelve subjects, recruited from the same pool as in previous experiments, participated in this experiment.

Apparatus and Stimuli. The apparatus and stimuli were the same as in the vertical/horizontal task of Experiment $2 \mathrm{~A}$.

Design. The design was the same as in Experiment 2A, except that only neutral flankers were used in this experiment.
}

\section{Results and Discussion}

The analysis of the results was done in the following way. For a vertical-line target (which required a rightkey response), a diagonal line tilted to the right was considered as a response-compatible flanker, and a diagonal line tilted to the left was considered as a responseincompatible flanker. The reverse was true for a horizontal line target. ${ }^{3}$ In the near conditions, the mean RT for a target with a response-compatible flanker was $492 \mathrm{msec}$, and the mean RT for a target with a response-incompatible flanker was $490 \mathrm{msec}$. In the far conditions, the mean RT for a target with a response-compatible flanker was $487 \mathrm{msec}$, and the mean RT for a target with a responseincompatible flanker was 490 msec. A 2 (response bias: compatible, incompatible) $\times 2$ (distance: near, far) ANOVA was performed. None of the effects were significant. Clearly, the diagonal-line flankers do not induce a response bias toward either the right response key or the left response key.

\section{EXPERIMENT 3}

One possible explanation for the results concerns the absolute RT of the subjects in the previous experiments. In all of the experiments reported so far, the subjects were faster in the congruent conditions of the diagonal task than in the congruent conditions of the vertical/horizontal task. Perhaps the congruency effect is smaller when the overall RT is slower. A careful look at the results of all of the experiments suggests that this explanation is not fully adequate. For example, the subjects in the vertical/ horizontal task of Experiment 2B (Figure 4) were as slow as the subjects in Experiment 1 for the same task (Figure 2), and yet the congruency effect was present in the former but not in the latter task. ${ }^{4}$ However, these comparisons are between subjects, and we may not have sufficient statistical power in the current experiments to rule out differences in absolute RT between the tasks.

In Experiment 3, we tested this explanation within a task by manipulating the luminance level of the stimuli. One group of subjects participated in this experiment. We used the diagonal task of Experiment 2A, with one alteration. Two different luminance levels were used. We expected the overall RT to be affected by this manipulation and to be slower in the dimmer condition. According to the hypothesis just described, we should also find a much reduced congruency effect in the dimmer condition. 


\section{Method}

Subjects. Sixteen subjects, drawn from the same pool as in the previous experiments, participated in this experiment.

Apparatus and Stimuli. The apparatus and stimuli were identical to those used in the diagonal task of Experiment 2A, except that two different luminance levels were used. Stimuli in the bright condition were identical to those used in the previous experiments $\left(76.6 \mathrm{~cd} / \mathrm{m}^{2}\right)$. Stimuli in the dim condition were presented at a luminance level of $1.5 \mathrm{~cd} / \mathrm{m}^{2}$. Both the asterisk that marked the location of the target and the error message displayed after incorrect responses had an intermediate luminance level of $29.5 \mathrm{~cd} / \mathrm{m}^{2}$.

Design. The design was essentially the same as the one used for the diagonal condition of Experiment 2A, except that half of the trials in each condition employed dim stimuli and the other half employed bright stimuli. The order of the trials within a block was random.

Procedure. The procedure was identical to that used in the previous experiments.

\section{Results and Discussion}

The correct RTs of the subjects in the bright condition are shown in the top panel of Figure 5. The bottom panel displays the results of the dim condition. As before, for each subject, we eliminated responses that were slower than $3 S D$ s above the mean. Two findings are quite clear from these results. First, the luminance manipulation was effective in elevating the subjects' RTs for the dim trials.
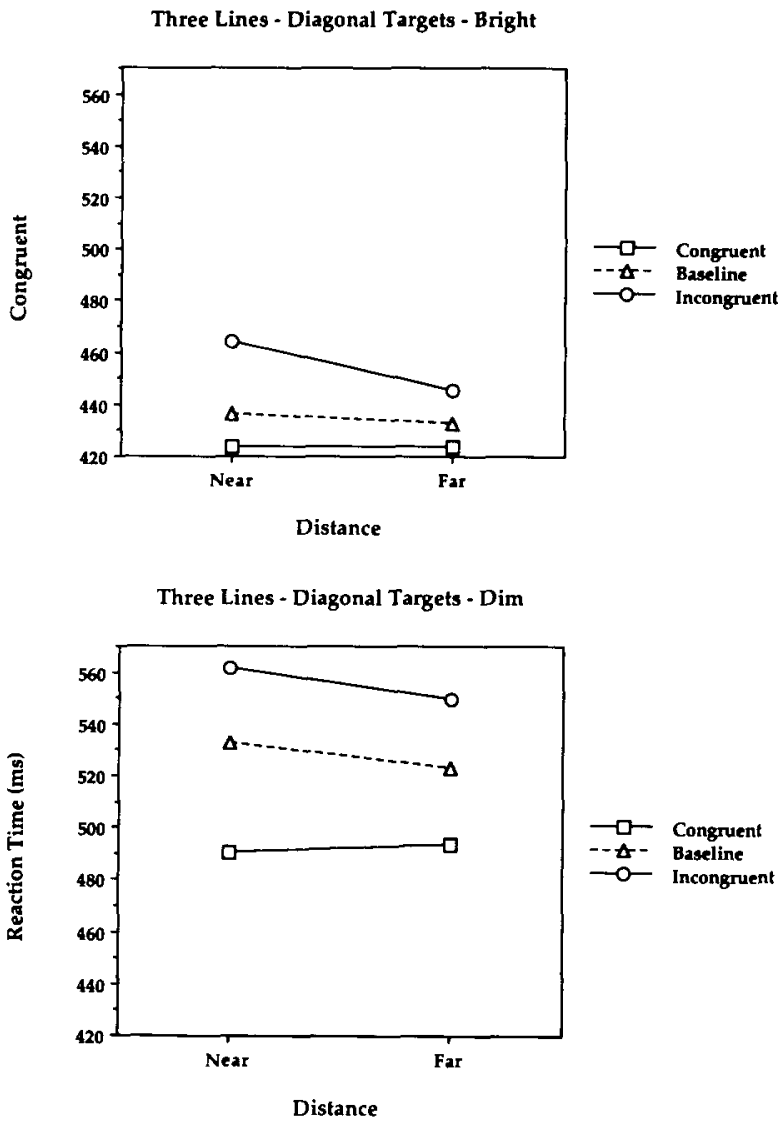

Figure 5. Mean RTs for the five congruency conditions in the diagonal task of Experiment 3. The top panel shows the RTs of the bright conditions, and the bottom panel shows the RTs of the dim conditions.
Second, the congruency effect was not reduced with slower RT. If anything, it was enhanced.

A 2 (luminance: dim, bright) $\times 3$ (congruency: congruent, neutral, incongruent) $\times 2$ (distance: near, far) repeated measures ANOVA was performed. The effect of brightness was significant $[F(1,15)=55.8, p<.05]$, indicating that subjects were reliably slower in the dim condition. Similar to our previous experiments with the diagonal task, the effects of congruency and distance were significant at $p<.05$, as was the interaction between them $[F(2,30)=159.8, F(1,15)=20.7, F(2,30)=$ 38.1 , for congruency, distance, and congruency $\times$ distance, respectively]. The only other significant effect was the interaction of brightness $\times$ congruency $[F(2,30)=$ $37.8, p<.05]$. This interaction was presumably due to a larger congruency effect in the dim condition. The results of this experiment rule out a direct relation between overall RT and the congruency effects obtained with the line stimuli.

\section{EXPERIMENT 4}

Treisman and Gormican (1988), using a visual search paradigm, have previously reported an asymmetry between line orientations. In their method, subjects had to search for the presence of a target among a variable number of distractors. The target appeared only in half of the trials, and its location was not known to the subjects in advance. Treisman and Gormican, as is common in this paradigm, were particularly interested in the relation between RT and the number of distractors present in the visual field (the array-size slope). They found that the array-size slope for a target tilted $18^{\circ}$ from the vertical frame of reference among vertical-line distractors was much more pronounced than was the array-size slope for a vertical-line target among tilted-line distractors.

The difference in angular orientation between the lines used in our study is much larger. Is there a relation between the asymmetry found by Treisman and Gormican (1988) and the one found in this study? An answer to this question could provide an additional constraint on the nature of the asymmetry between the diagonal lines and the vertical/horizontal lines used in our study. We examined this issue by using our stimuli in a visual search paradigm similar to the one employed by Treisman and Gormican.

\section{Method}

Subjects. Twenty-four subjects, drawn from the same pool as in the previous experiments, participated in this experiment. They were divided into four groups of 6 subjects. Each group participated in a different condition.

Apparatus and Stimuli. The apparatus was identical to that of previous experiments. The stimuli consisted of four types of line orientation: horizontal, vertical, diagonal tilted $45^{\circ}$ to the right, and diagonal tilted $45^{\circ}$ to the left. The length of all stimuli was $0.37^{\circ}$, and their luminance was the same as in Experiment 1 .

Design. All four groups of subjects were instructed to search for a target among a variable number of distractors. The target for one group was a diagonal line tilted to the right. The target for a second group was a diagonal line tilted to the left. The distractors for 
both of these groups consisted of either horizontal or vertical lines (but never both), and the type of distractor used on each trial was determined randomly. The third group of subjects searched for a vertical-line target, and the fourth group searched for a horizontalline target. The distractors for these last two groups consisted of either diagonal lines tilted to the right or to the left. Again, the type of distractor used on each trial was selected randomly.

The specific design we used is similar to the one used previously in our lab (e.g., Cohen \& Ivry, 1991). Each display in this experiment consisted of either $4,8,16$, or 24 items. The different array sizes were mixed within a block. Half of the trials in each array-size condition included the target, and the remaining half included only distractors. The stimuli on each trial were positioned on two imaginary circles. The radii of the inner and outer circles extended approximately $2.2^{\circ}$ and $3.3^{\circ}$ from the center. For array sizes of 4 and 8 items, all of the stimuli were located on either the inner or the outer circle, which was randomly selected. For array sizes of 16 and 24 items, half of the items were located on each circle. The minimal distance between adjacent items was approximately $1.1^{\circ}$.

The subjects received a short practice block of 24 trials, followed by five experimental blocks of 80 trials each with 10 trials per condition, for a total of 400 experimental trials.

Procedure. At the beginning of each trial, an asterisk, serving as a fixation point, was presented on the center of the visual screen. After $1,000 \mathrm{msec}$, the asterisk was replaced by the stimulus display. The subjects were required to push the left key on the twokey device if the target was present and to push the right key if no target was present. An error message was presented on the screen for $500 \mathrm{msec}$ following incorrect responses. The subjects were instructed to expect occasional errors because of the emphasis on speeded responses. The screen went blank immediately following the subject's correct response until the appearance of the asterisk of the next trial. The ITI was $2,000 \mathrm{msec}$.

\section{Results and Discussion}

A preliminary ANOVA did not reveal any difference between the two groups of subjects who searched for either a vertical- or a horizontal-line target. Similarly, there was no difference between subjects who searched for a left diagonal target and those who search for a right diagonal target. Therefore, we combined the data for the two diagonal targets, and we also combined the data for vertical and horizontal targets. The RTs of the subjects are shown in Figure 6. Only correct responses are included. The top panel shows the results for the targetpresent condition, and the bottom panel shows the results for the target-absent trials. Several observations are evident from the results. First, the RTs in the target-present condition were not affected by array size in either the diagonal tasks or the vertical and horizontal tasks. Indeed, the estimated slope by a regression analysis was slightly less than 0 for all tasks. Second, the overall RTs in the diagonal tasks were faster than those in the vertical and horizontal tasks. The estimated intercept is $525 \mathrm{msec}$ for the two diagonal tasks and $615 \mathrm{msec}$ for the vertical and horizontal tasks. Third, array size had a small effect on the RTs in the target-absent condition. Fourth, the arraysize slope in the target-absent condition was more pronounced in the vertical and horizontal tasks (estimated slope, $4.5 \mathrm{msec}$ ) than in the diagonal tasks (estimated slope, $1.5 \mathrm{msec}$ ).

These observations were basically confirmed by a mixed design ANOVA. The results were submitted to a 2 (task:
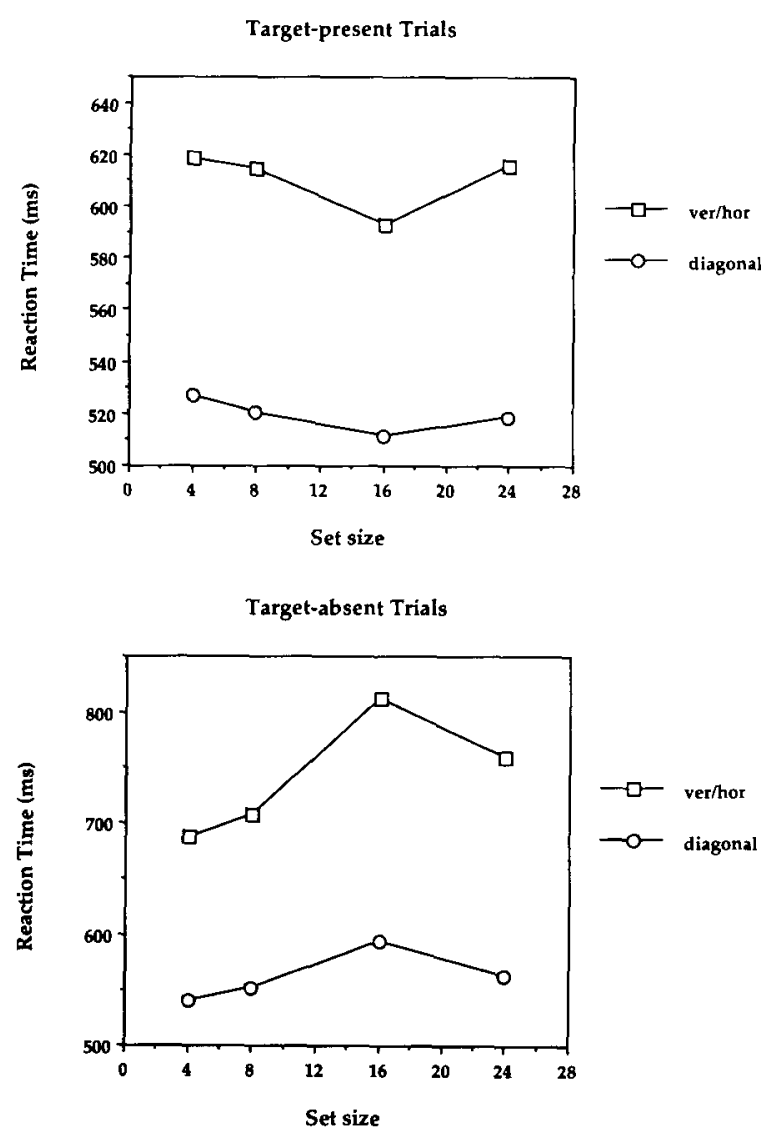

Figure 6. Mean RTs of the visual search for the diagonal targets and for the vertical/horizontal targets of Experiment 4. The top panel shows the RTs for the target-present condition, and the bottom panel shows the RTs for the target-absent trials.

vertical/horizontal, diagonal) $\times 2$ (target: present, absent) $\times 4$ (array size: $4,8,16,24)$ analysis. All the main effects were significant at $p<.05[F(1,22)=9.3$, $F(1,22)=27.7$, and $F(3,66)=10.7$, for the main effects of task, target, and array size, respectively]. The task $\times$ target interaction was significant $[F(1,22)=6.8]$, indicating that the overall RT difference between the diagonal and vertical/horizontal tasks was more pronounced in the target-absent condition. The target $\times$ set size interaction was significant as well $[F(3,66)=15.8]$, because the array-size slope was evident only in the target-absent condition. Finally the task $\times$ array size and the triple interaction were just marginally significant $[F(3,66)=2.7$, $p<.06$, and $F(3,66)=2.5, p<.07$, for the task $\times$ array size and the triple interaction, respectively]. Thus, all the observations mentioned above were confirmed, except that the difference between the two tasks with regard to array-size slopes in the target-absent conditions was only marginally significant.

These results differ from those reported by Treisman and Gormican (1988). Although Treisman and Gormican found a sizable difference in the array-size slope between tilted and vertical lines for both the target-present and 
target-absent conditions, we did not find any difference in the slope for the target-present condition, and we found only a marginally significant difference for the targetabsent condition. We did find a difference in the intercept for diagonal lines versus vertical/horizontal lines, however. Treisman and Gormican reported a smaller intercept difference between the tilted and vertical stimuli in their experiment $(46 \mathrm{msec})$ but did not provide a statistical analysis for the reliability of this difference. Thus, we cannot say with any certainty whether the difference in the magnitude of the intercept between the experiments is reliable. It is clear, however, that detection of either vertical or horizontal lines among diagonal lines tilted $45^{\circ}$ is not affected at all by the number of distractors present in the scene. This finding suggests that search for vertical and horizontal lines is performed in parallel and without capacity limitation (Townsend, 1976, 1990).

\section{GENERAL DISCUSSION}

The experiments with the flanker task reported in this article suggest that there exists an asymmetry between diagonal lines and vertical/horizontal lines. When the target is either a vertical or a horizontal line, diagonal-line flankers have the same effect as incongruent flankers. In contrast, when the target is a diagonal line tilted either to the right or to the left, vertical- and horizontal-line flankers do not have the same effect as incongruent flankers.

The visual search experiment adds a constraint on this asymmetry. Although the detection of a vertical- or horizontal-line target among diagonal distractors requires more time than does the detection of a diagonal-line target among vertical/horizontal distractors (as was evidenced by the intercept difference between the two tasks), neither search is influenced by the number of distractors. There are good reasons to believe that, when the arraysize slope is flat, the difference between the target and the distractors can be detected outside of the central focus of attention (e.g., Shiffrin \& Schneider, 1977). Therefore, although the flanker-task experiments suggest that diagonal lines are incongruent with both vertical and horizontal lines, the visual search study suggests that the representation of diagonal lines outside of the central focus of attention is sufficiently distinct from either vertical or horizontal lines.

What is the reason for this asymmetry? First, a class of explanations based on feature interaction, called an "interactive channel" (e.g., Estes, 1972, 1974), can be ruled out. As pointed out by Estes (1982), the extent of the feature interaction in this class of models is a function of the distance between the objects. The effects in the present experiments were observed even when the distance between the flankers and the target exceeded $1^{\circ}$ of visual angle, which is far larger than the distances in which the feature interaction was observed by Estes (e.g., 1982).

One possible explanation for the results is an extension of the explanation suggested by Treisman and Gormican
(1988) to account for the asymmetries they observed between tilted and vertical lines. As mentioned above, Treisman and Gormican found that the array-size slope in visual search for a slightly tilted target among vertical distractors is less steep than the array-size slope for a vertical target among slightly tilted distractors. Treisman and Gormican suggested that tilted lines outside of the central focus of attention are represented as a deviation from vertical lines. That is, the vertical line serves as a reference point and tilted lines are represented as the amount of change from this reference point. Thus, in some sense, the representation of tilted lines includes the reference point plus the deviation from that point.

We can extend this suggestion to the present situation by assuming that each tilted line is defined with respect to both vertical and horizontal reference points. The closer the line is in angular rotation to one of the reference points, the stronger would be the association with that reference point. For example, a line tilted $15^{\circ}$ from the vertical meridian (and thus $75^{\circ}$ from the horizontal meridian) would have a stronger association with the vertical reference point than with the horizontal reference point. A line tilted $45^{\circ}$, as were the lines used in the present experiments, would have roughly equal associations with both reference points. Vertical (horizontal) lines are only associated with a vertical (horizontal) reference point.

This suggestion readily explains the results obtained with the flanker task. Diagonal lines, by the nature of their representation, activate both vertical- and horizontal-line representations. When vertical and horizontal lines are associated with competing responses, as was the case in the vertical/horizontal task, the presence of diagonal-line flankers presumably activates both targets and leads to response competition.

The flat array-size slopes obtained in the visual search experiment can also be explained by this hypothesis. When the target was not present in this experiment, all of the distractors were identical to each other. Thus, the task of the subjects was basically to detect whether one of the stimuli deviates from the others. When searching for a vertical (or a horizontal) line among diagonal distractors, the subjects had to detect whether one of the stimuli activated just the vertical (or horizontal) reference point. The results of the experiment suggest that subjects can perform this task quite effectively.

This hypothesis seems reasonable because it extends a previous hypothesis (by Treisman \& Gormican, 1988) and it can account qualitatively for the data. However, a closer examination of this explanation reveals some problems. As shown in Experiment 4, diagonal lines are not completely similar to horizontal or vertical lines. Thus, in Experiments 1 and 2, there should be more interference from incongruent flankers than from the neutral diagonal lines. Yet, the effect of the diagonal-line flankers was virtually the same as that of the incongruent flankers.

Another possible explanation is related to findings reported by O'Hara (1980; see also O'Hara \& C. W. Eriksen, 1979) concerning letter matching. These authors 
found that when subjects are required to judge whether two letters in known positions are the same, the appearance of letters (e.g., GDO) in irrelevant positions slows down the subjects' latencies. However, when the letters in the irrelevant positions form a word (e.g., GOD), they do not affect the subjects' latencies. One interpretation of these findings is that when the irrelevant stimuli are represented at the same level of representation as the relevant stimuli (letters in the example above), they cause interference. But when the irrelevant stimuli are represented at a different level of representation from that of the relevant stimuli (letters and words in the example), they do not cause any interference. It is possible that vertical and horizontal lines have a special status in the representation of line orientations. Thus, when neutral vertical or horizontal lines are presented in irrelevant positions, subjects can ignore them (just as they could ignore words in letter matching). However, when neutral diagonal lines appear in irrelevant positions, subjects cannot ignore them.

The two explanations outlined here are clearly incomplete. More research is needed to decide between these hypotheses and others that were not mentioned. Although the nature of the asymmetry in orientation is still speculative, the present study does constrain its nature. The experiments indicate that it is due to the nature of the representations of line orientations outside of the central focus of attention and is not the result of the specific method we employed in our experiments.

The present study implies a second, more general point. The experiments demonstrate that perceptual relations between the target and the flankers can have more complex effects on congruency than were previously envisioned. These possible effects of structural relations between stimuli have to be taken into account in studies using the flanker task.

Finally, since the present study adds constraints to the nature of early representations of line orientations outside of the central focus of attention, experiments using this method can also shed light on the nature of other representations outside of the focus of attention. For example, Tsal and Mevorach (1991) have recently shown that the length of such stimuli is consistently judged to be greater than the length of the same stimuli when attention is directed toward them. It is possible, therefore, that in a flanker task in which responses are determined by the length of the target, flankers will be congruent with the target only when they are shorter than the target.

\section{REFERENCES}

Brondbent, D. E. (1958). Perception and communication. London: Pergamon.

CoHEN, A., \& IVRY, R. (1991). Density effects in conjunction search: Evidence for a coarse location mechanism of feature integration. Journal of Experimental Psychology: Human Perception \& Performance, 17, 891-901.
Coles, M. G. H., Gratton, G., Bashore, T. R., Eriksen, C. W., \& DONChIN, E. (1985). A psychophysiological investigation of the continuous flow model of human information processing. Journal of Experimental Psychology: Human Perception \& Performance, 11, 529-553.

Deutsch, J. A., \& Deutsch, D. (1963). Attention: Some theoretical considerations. Psychological Review, 70, 80-90.

Driver, J., \& BAyLIS, G. C. (1989). Movement and visual attention: The spotlight metaphor breaks down. Journal of Experimental Psychology: Human Perception \& Performance, 15, 448-456.

Eriksen, B. A., ERIKSEN, C. W. (1974). Effects of noise letters upon the identification of a target letter in a nonsearch task. Perception \& Psychophysics, 16, 143-149.

ERIKSEN, C. W., ERIKSEN, B. A. (1979). Target redundancy in visual search: Do repetitions of the target within the display impair processing? Perception \& Psychophysics, 26, 195-205.

ErIKSEN, C. W., \& Schultz, D. W. (1979). Information processing in visual search: A continuous flow conception and experimental results. Perception \& Psychophysics, 25, 249-263.

EsTES, W. K. (1972). Interactions of signal and background variables in visual processing. Perception \& Psychophysics, 12, 278-286.

EsTES, W. K. (1974). Redundancy of noise elements and signals in visual detection of letters. Perception \& Psychophysics, 16, 53-60.

EsTES, W. K. (1982). Similarity-related channel interactions in visual processing. Joumal of Experimental Psychology: Human Perception \& Performance, 8, 353-382.

Grice, G. R., \& GWYNNE, J. W. (1985). Temporal characteristics of noise conditions producing facilitation and interference. Perception \& Psychophysics, 37, 495-501.

Harms, L., Bundesen, C. (1983). Color segregation and selective attention in a nonsearch task. Perception \& Psychophysics, 33, 11-19.

Kramer, A. F., \& Jacobson, A. (1991). Perceptual organization and focused attention: The role of objects and proximity in visual processing. Perception \& Psychophysics, 50, 267-284.

Kramer, A. F., Tham, M.-P., \& YeH, Y.-Y. (1991). Movement and focused attention: A failure to replicate. Perception \& Psychophysics, 50, 537-546.

Miller, J. (1987). Priming is not necessary for selective-attention failures: Semantic effects of unattended, unprimed letters. Perception \& Psychophysics, 41, 419-434.

MiLLER, J. (1991). The flanker compatibility effect as a function of visual angle, attentional focus, visual transients, and perceptual load: $A$ search for boundary conditions. Perception \& Psychophysics, 49, 270-288.

O'HaRa, W. P. (1980). Evidence in support of word unitization. Perception \& Psychophysics, 27, 390-402.

O'HarA, W., \& ERIKSEN, C. W. (1979). Word unitization examined using an interference paradigm. Bulletin of the Psychonomic Society, 14, 81-84.

SHIFFRIN, R. M., \& SCHNEIDER, W. (1977). Controlled and automatic human information processing: II. Perceptual learning, automatic attending, and a general theory. Psychological Review, 87, 127-190.

TownSEND, J. T. (1976). Serial and within-stage independent parallel model equivalence on the minimum completion time. Joumal of Mathematical Psychology, 14, 219-239.

TownsEnd, J. T. (1990). Serial vs. parallel processing: Sometimes they look like tweedledum and tweedledee but they can (and should) be distinguished. Psychological Science, 1, 46-54.

TreismaN, A. M., \& GormicaN, S. (1988). Feature analysis in early vision: Evidence from search asymmetries. Psychological Review, 95 , $15-48$.

Tsal, Y., \& Mevorach, L. (1991, November). Effects of attention on length and distance estimates. Paper presented at the 32nd Annual Meeting of the Psychonomic Society, San Francisco.

Yantis, Y., \& Johnston, J. C. (1990). On the locus of visual selection: Evidence from focused attention tasks. Journal of Experimental Psychology: Human Perception \& Performance, 16, 135-149.

YEH, Y.-Y., \& ERIKSEN, C. W. (1984). Name codes and features in the discrimination of letter forms. Perception \& Psychophysics, 36, 225-233. 


\section{NOTES}

1. Kramer, Tham, and Yeh (1991) have recently reported a series of experiments in which they failed to replicate the results of Driver and Baylis (1989). We have also conducted a number of experiments in an effort to replicate these findings. As with Kramer et al., we were unsuccessful.

2. The size manipulation was originally designed for purposes not discussed in this article. One consequence of the original design is that the length of the neutral stimuli was not manipulated. In addition, the physical characteristics of our monitor did not enable us to create diagonal lines of the exact same lengths as the vertical and horizontal lines. However, as discussed later in the text, the size manipulation had no effect on the results of any of the experiments reported in this study. Therefore, it is unlikely that either the differences in size between the two tasks or the fixed size of the neutral stimulus had any effect on the results.
3. It is possible that a right-tilted line may induce a response bias toward the left key (because its lower part points to the left) and that a left-tilted line may induce a response bias toward the right key. The findings of no response bias rule out this possibility as well.

4. Comparison of the flanker task between studies immediately reveals that the congruency effect can be obtained over a range of RTs (compare, for example, B. A. Eriksen \& C. W. Eriksen, 1974, with Miller, 1991). However, these experiments never compared two specific stimuli, such as diagonal and vertical/horizontal lines, over a range of RTs. It is possible that the congruency effect for a given stimulus changes with the overall RT.

(Manuscript received June 3, 1992; revision accepted for publication December 9, 1992.)

\section{Erratum}

Fortin, C., Rousseau, R., Bourque, P., and Kirouac, E. Time estimation and concurrent nontemporal processing: Specific interference from short-term-memory demands. Perception \& Psychophysics, 1993, 53(5), 536-548-On page 546, what should have been the sixth line in column 2 (first line of paragraph 2) is missing. With the missing line inserted, paragraph 2 should correctly start out with:

Task complexity refers to the number of operations re-

and continue with:

quired to accomplish a task, and task difficulty refers to 